\title{
Backreaction due to inhomogeneities and the future evolution of an accelerating universe
}

\section{Majumdar A.S., Bose N.}

S. N. Bose National Centre for Basic Sciences, Block JD, Sector III, Salt Lake, Kolkata, India;

E-mail: Majumdar<archan@bose.res.in>;

We investigate the effect of backreaction due to inhomogeneities on theevolution of the present universe by considering a multi-scale model within the Buchert framework. Taking the observed present acceleration of the universe as an essential input, we study the effect of inhomogeneities in the future evolution. We find that the backreaction from inhomogeneities causes the acceleration to slow down in the future for a range of initial configurations and model parameters, and even lead in certain cases to the emergence of a future decelerating epoch.

Keywords: Dark energy, Cosmic backreaction, Large scale structure.

DOI: $10.18698 / 2309-7604-2015-1-365-373$

\section{Introduction}

By now the present acceleration of the Universe is quite well established observationally [1]. The cause for it is attributed to a mysterious component called Dark Energy whose true nature is still unknown to us, although there is no dearth of innovative ideas to ac-count for the present acceleration [2]. In recent times there is an upsurge of interest on studying the effects of inhomogeneities on the expansion of the Universe and several approaches have been developed to facilitate this [3-8]. It has been argued [7] that backre-action from inhomogeneities from the era of structure formation could lead to an accelerated expansion of the Universe.

The Buchert framework $[4,5]$ for evaluating the effect of backreaction on the global metric has been further extended in [9] where the universe is considered to be divided into multiple domains and subdomains. The model considered in [9] involved the simplification of clubbing together all spatial domains into one overdense subdomain and another underdense subdomain. Recently, we have studied the backreaction scenario within the Buchert framework using a simple two-scale model [10], which shows the possibility of the global acceleration disappearing in the future as a result of backreaction due to inho-mogeneities. Consideration of an explicit event horizon associated with the era of present acceleration favours further the possibility of a transition to a future decelerated era [11]. In the present work we try to improve upon this model to mimic the real universe to a closer extent. Instead of the previous two-scale model $[9,10,11]$, here we consider the Universe as a global domain D which is partitioned into multiple overdense and underdense regions, and all which are taken to evolve differently from each other. 


\section{The Backreaction Framework}

In the framework developed by Buchert $[4,5,12]$ for a compact spatial domain $\mathcal{D}$ the scalefactor $a_{\mathscr{D}}(t)=\left(\frac{|\mathcal{D}|_{g}}{\left|\mathcal{D}_{i}\right|_{g}}\right)^{1 / 3}$, encodes the average stretch of all directions of the domain where $|\mathcal{D}|_{g}$ is the volume of $\mathscr{D}$. Using the Einstein equations, with a pressure-less fluid source, we get the following equations $[4,5,12]$

$$
\begin{gathered}
3 \frac{\ddot{a}_{\mathscr{D}}}{a_{\mathscr{D}}}=-4 \pi G\langle\rho\rangle_{\mathscr{D}}+Q_{\mathscr{D}}+\Lambda \\
3 H_{\mathscr{D}}^{2}=8 \pi G\langle\rho\rangle_{\mathscr{D}}-\frac{1}{2}\langle R\rangle_{\mathscr{D}}-\frac{1}{2} Q_{\mathscr{D}}+\Lambda \\
0=\partial_{t}\langle\rho\rangle_{\mathscr{D}}+3 H_{\mathscr{D}}\langle\rho\rangle_{\mathscr{D}}
\end{gathered}
$$

Here the average of the scalar quantities on the domain $\mathcal{D}$ is defined as, $\langle f\rangle_{\mathcal{D}}(t)=\frac{\int_{\mathcal{D}} f\left(t, X^{1}, X^{2}, X^{3}\right) d \mu_{g}}{\int_{\mathscr{D}} d \mu_{g}}=|\mathcal{D}|_{g}^{-1} \int_{\mathcal{D}} f d \mu_{g}$ and where $\rho, \mathcal{R}$ and $H_{\mathcal{D}}$ denote the local matter density, the Ricci-scalar of the three metric $g_{i j}$, and the domain dependent Hubble rate $H_{\mathcal{D}}=\dot{a}_{\mathcal{D}} / a_{\mathcal{D}}$ respectively. The kinematical backreaction $\mathcal{Q}_{\mathcal{D}}$ is defined as $Q_{\mathscr{D}}=\frac{2}{3}\left(\left\langle\theta^{2}\right\rangle_{\mathscr{D}}-\langle\theta\rangle_{\mathscr{D}}^{2}\right)-2 \sigma_{\mathscr{D}}^{2}$ here is the local expansion rate and $\sigma^{2}=1 / 2 \sigma_{i j} \sigma^{i j}$ is the squared rate of shear.

The "global" domain $\mathcal{D}$ is assumed to be separated into subregions, such that $|\mathcal{D}|_{g}=\sum_{\ell}\left|\mathcal{F}_{\ell}\right|_{g}$, where $\mathcal{F}_{\ell}$ denotes a subregion. The expression relating the acceleration of the global domain to that of the sub-domains is given by [9]: 


$$
\frac{\ddot{a}_{\mathcal{D}}}{a_{\mathcal{D}}}=\sum_{\ell} \lambda_{\ell} \frac{\ddot{a}_{\ell}(t)}{a_{\ell}(t)}+\sum_{\ell \neq m} \lambda_{\ell} \lambda_{m}\left(H_{\ell}-H_{m}\right)^{2}
$$

where $\lambda_{\ell}=\left|\mathcal{F}_{\ell}\right|_{g} /|\mathcal{D}|_{g}$ is the volume fraction of the subregion $\mathcal{F}_{\ell}$, with $\sum_{i} \lambda_{i}=1$

\section{Future Evolution for the presently accelerating universe}

We will now explore the future evolution of the Universe after the current stage of acceleration sets in. We consider $\mathcal{D}$ to be partitioned into equal numbers of overdense and underdense sub-domains. We label all overdense sub-domains by $\mathcal{M}$ (called 'Wall') and all underdense domains as $\mathcal{E}$ (called 'Void'), such that $\mathcal{D}=\left(\cup_{j} \mathcal{M}^{j}\right) \cup\left(\cup_{j} \mathcal{E}^{j}\right)$.We assume that the scale-factors of the regions $\mathcal{E}^{j}$ and $\mathcal{M}^{j}$ are, respectively, given by $a_{\mathcal{E}_{j}}=c_{\mathcal{E}_{j}} t^{\alpha_{j}}$ and $a_{\mathcal{M}_{j}}=c_{\mathcal{M}_{j}} t^{\beta_{j}}$ where $\alpha_{j}, \beta_{j}, c_{\varepsilon_{j}}$ and $c_{\mu_{j}}$ are constants. The volume fraction of the subdomain $\mathcal{M}^{j}$ is given by $\lambda_{\mathcal{M}_{j}}=\frac{\left|\mathcal{M}^{j}\right|_{g}}{|\mathcal{D}|_{g}}$, which can be rewritten in terms of the corresponding scale factors as $\lambda_{\mathcal{M}_{j}}=\frac{a_{\mathcal{M}_{j}}^{3}\left|\mathcal{M}_{i}^{j}\right|_{g}}{a_{\mathcal{D}}^{3}\left|\mathcal{D}_{i}\right|_{g}}$, and similarly for the $\mathcal{E}^{j}$ subdomains. We thereforefind that the global acceleration equation (4) becomes

$$
\begin{aligned}
& \frac{\ddot{a}_{\mathscr{D}}}{a_{\mathscr{D}}}=\sum_{j} \frac{g_{\mathcal{M}_{j}}^{3} t^{3 \beta_{j}}}{a_{\mathscr{D}}^{3}} \frac{\beta_{j}\left(\beta_{j}-1\right)}{t^{2}}+\sum_{j} \frac{g_{\varepsilon_{j}}^{3}}{a_{\mathscr{D}}^{3}} \frac{\alpha_{j}\left(\alpha_{j}-1\right)}{t^{2}} \\
& +\sum_{j \neq k} \frac{g_{\mathcal{M}_{j}}^{3} t^{3 \beta_{j}}}{a_{\mathscr{D}}^{3}} \frac{g_{\mathscr{M}_{k}}^{3} t^{3 \beta_{k}}}{a_{\mathscr{D}}^{3}}\left(\frac{\beta_{j}}{t}-\frac{\beta_{k}}{t}\right)^{2} \\
& +\sum_{j \neq k} \frac{g_{\varepsilon_{j}}^{3} t^{3 \alpha_{j}}}{a_{\mathscr{D}}^{3}} \frac{g_{\varepsilon_{k}}^{3} t^{3 \alpha_{k}}}{a_{\mathscr{D}}^{3}}\left(\frac{\alpha_{j}}{t}-\frac{\alpha_{k}}{t}\right)^{2} \\
& +2 \sum_{j, k} \frac{g_{\mathcal{M}_{j}}^{3} t^{3 \beta_{j}}}{a_{\mathscr{D}}^{3}} \frac{g_{\varepsilon_{k}}^{3} t^{3 \alpha_{k}}}{a_{\mathscr{D}}^{3}}\left(\frac{\beta_{j}}{t}-\frac{\alpha_{k}}{t}\right)^{2}
\end{aligned}
$$


where $g_{\mathcal{M}_{j}}^{3}=\frac{\lambda_{\mathcal{M}_{j 0}} a_{\mathscr{D}_{0}}^{3}}{t_{0}^{3 \beta_{j}}}$ and $g_{\varepsilon_{j}}^{3}=\frac{\lambda_{\varepsilon_{j 0}} a_{\mathscr{D}_{0}}^{3}}{t_{0}^{3 \alpha_{j}}}$ are constants.

We now perform a comparative study of the two cases where (i) the global domain $\mathcal{D}$ is considered to be divided into 50 overdense and underdense subdomains each, and (ii) 100 overdense and underdense subdomains each. In order to obtain numerical solutions of equation (5) we willconsider the range of values for the parameters $\alpha_{j}$ and $\beta_{j}$ as a Gaussian distribution, which is of the form $\frac{1}{\sigma \sqrt{2 \pi}} \exp \left(-\frac{(x-\mu)^{2}}{2 \sigma^{2}}\right)$, where $\sigma$ is the standard deviation and is the mean (therange of values corresponds to the full width at half maximum of thedistribution). We also assign values for the volume fractions $\lambda_{\mathcal{M}_{j}}$ and $\lambda_{\mathcal{E}_{j}}$ based on a Gaussian distribution and impose the restriction that the totalvolume fraction of all the overdense subdomains at present time should be 0.09 , a value that has been determined through numerical simulationsin the literature [9]. Note here that using our ansatz for the subdomain scale factors one may try to determine theglobal scale factor through Eq. (4). In order to doso, one needs to know the initial volume fractions $\lambda_{I_{i}}$ which are in turn related to the $c_{\mathcal{E}_{j}}$ and $c_{\mathcal{M}_{j}}$. However, in our approach based upon the Buchert framework $[4,5,12]$ we do not need to determine $c_{\mathcal{E}_{j}}$ and $c_{\mathcal{M}_{j}}$, but instead, obtain from Eq.(5) the global scale factornumerically by the method of recursive iteration, using as an 'initialcondition' the observational constraint $q_{0}=-0.7$, where $q_{0}$ is the current value of the deceleration parameter. The expression for $q_{0}$ is a completely analytic function of $\alpha_{j}, \beta_{j}$ and $t_{0}$, but since we are studying the effect of inhomogeneitiestherefore the Universe cannot strictly be described based on a FRWmodel and hence the current age of the Universe $\left(t_{0}\right)$ cannot be fixed based on current observations which use the FRW model tofix the age. Instead for each combination of values of the parameters $\alpha_{j}$ and $\beta_{j}$ we find out the value of $t_{0}$ for our model from (5) by taking $q_{0}=-0.7$. 


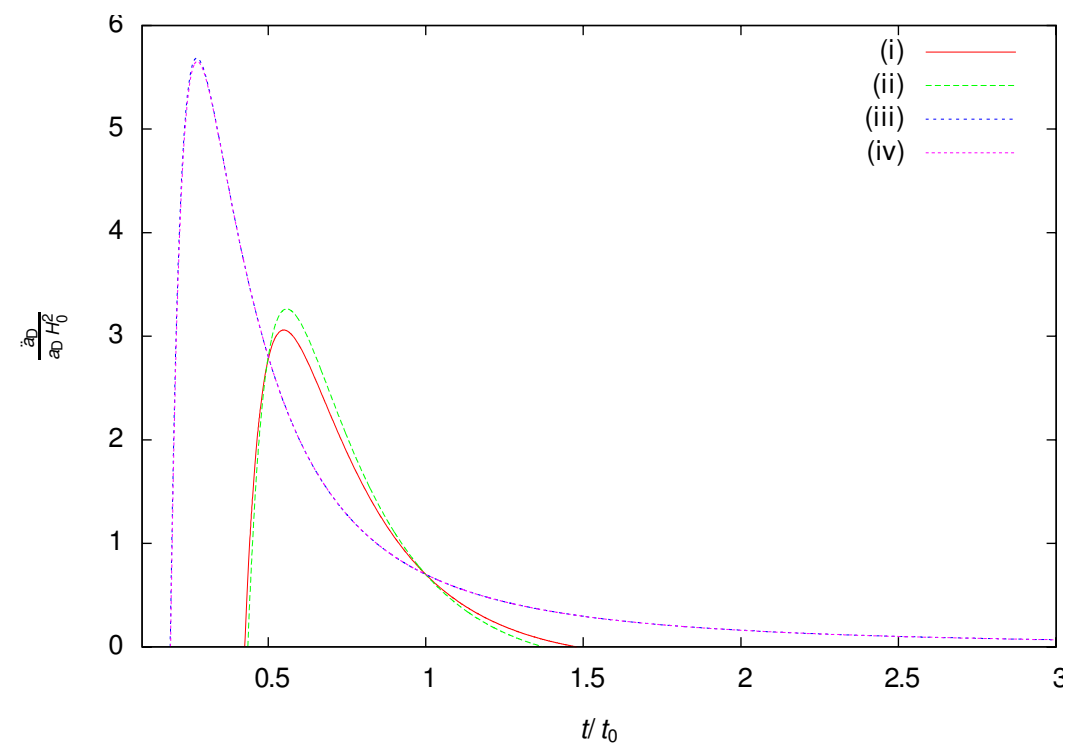

Fig. 1. The dimensionless global acceleration parameter $\frac{\ddot{a}_{\mathcal{D}}}{a_{\mathcal{D}} H_{0}^{2}}$, plotted vs. time (in units of $t / t_{0}$ with $t_{0}$ being the current age of the Universe). In curves (i) and (ii) the value of $\alpha$ is in the range $0.990-0.999$ and that of $\beta$ is in the range $0.58-0.60$. In curves (iii) and (iv) the value of $\alpha$ is inthe range $1.02-1.04$ and that of $\beta$ is in the range $0.58-0.60$

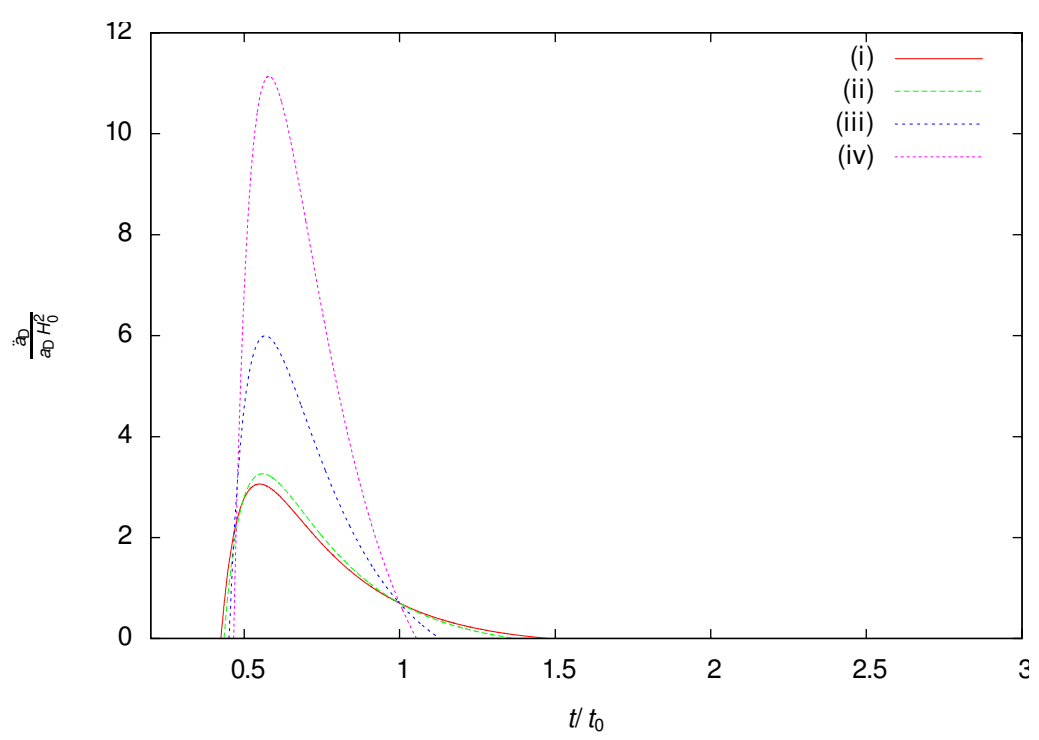

Fig. 2. Here also $\frac{\ddot{a}_{D}}{a_{D} H_{0}^{2}}$ is plotted vs. time. In curves (i) and (ii) the value of $\alpha_{\alpha}$ lies in the range $0.990-0.999$ and that of $\beta$ is in the range $0.58-0.60$. For curves (iii) and (iv) the value of ${ }_{\alpha}$ is in the range $0.990-0.999$ and that of $\beta$ is in the range $0.55-0.65$ 
The global acceleration for the two cases has been plotted in Figs. 1 and 2. In both the figures, the curves (i) and (iii) are for the case where the global domain $\mathcal{D}$ is partitioned into 50 overdense and underdense subdomains each, and the curves (ii) and (iv) correspond to the case where $\mathcal{D}$ is partitioned into 100 overdense and underdense subdomains each. Thevalues for the expansion parameters $\beta_{j}$ of the overdense subdomains is taken to lie between $1 / 2$ and $2 / 3$ since the expansionis assumed to be faster than in the radiation dominated case, andis upper limited by the value for matter dominated expansion. In Fig. 1 the behaviour of global acceleration is shown for values of $\alpha_{j}<1$ and also $\alpha_{j}>1$, keeping the range of values of $\beta_{j}$ quite narrow and also the same for all four curves. We have kept the value of $\alpha_{j}$ close to 1 when $\alpha_{j}<1$ because if $\alpha_{j}$ is less than a certain value, which depends on the value of $\beta_{j}$, then the acceleration becomes undefined aswe do not get real solutions from (5). In order to demonstratethis fact analytically let us consider a toy model where $\mathcal{D}$ is divided into one overdense subdomain $\mathcal{M}$ and one underdense subdomain $\mathcal{E}$. Using the fact that $\lambda_{\mathcal{M}}+\lambda_{\mathcal{E}}=1$, Eq. (5) can be written as

$$
\begin{aligned}
\frac{\ddot{a}_{\mathcal{D}}}{a_{\mathcal{D}}} & =\frac{g_{\mathcal{M}}^{3} t^{3 \beta}}{a_{\mathcal{D}}^{3}} \frac{\beta(\beta-1)}{t^{2}}+\left(1-\frac{g_{\mathcal{M}}^{3} t^{3 \beta}}{a_{\mathcal{D}}^{3}}\right) \frac{\alpha(\alpha-1)}{t^{2}} \\
& +2 \frac{g_{\mathcal{M}}^{3} t^{3 \beta}}{a_{\mathcal{D}}^{3}}\left(1-\frac{g_{\mathcal{M}}^{3} t^{3 \beta}}{a_{\mathcal{D}}^{3}}\right)\left(\frac{\beta}{t}-\frac{\alpha}{t}\right)^{2}
\end{aligned}
$$

This shows us that we get real time solutions for $\alpha \geq \frac{1}{3}[(\beta+1)+2 \sqrt{2 \beta(1-\beta)}]$. If we now consider $\beta=0.5$ (its lowest possible value) then we get $\alpha \geq 0.971404521$ and if we consider $\beta=0.66$ (itshighest possible value) then we get $\alpha \geq 0.999950246$. Henceas stated earlier, for a particular value of $\beta$ we have a lower limit on the value of $\alpha$.

In Fig. 1, for $\alpha_{j}<1$ the acceleration becomes negative inthe future for both cases of partitioning (curves (i) and (ii)). The acceleration reaches a greater value and at a slightly later time when $\mathcal{D}$ is partitioned into 100 overdense and underdense subdomains (curve (ii)) and also becomes negative at an earlier timeas compared to the case where $\mathcal{D}$ is partitioned into 50 overdense and underdense 
subdomains (curve (i)). When $\alpha_{j}>1$ then we see that the acceleration curves for the two cases are almost identical, with the maximum value being very slightly larger for partition type (i) (curve (iii)). After reaching the maximum the acceleration decreases and goes asymptotically to a small positive value. When $\alpha_{j}<1$ then the first two terms of (5) are negative, but the last term, which is always positive, gains prominence as the number of subdomains increases thus increasing the acceleration. When $\alpha_{j}>1$ then only the first term in (5) is negativeand hence the acceleration curves for the two partition cases (curves (iii) and (iv)) are very similar, the only visible difference being the slightly higher maximum value when $\mathcal{D}$ is partitioned into a lower number of subdomains.

In Fig. 2 we have illustrated the behaviour of the global accelerationby taking narrow and broad ranges of values of $\beta_{j}$ and keeping $\alpha_{j}<1$ and the same for all the curves. As seen in Fig. 1here also the acceleration becomes negative in the future for all the curves because we have $\alpha_{j}<1$ for all of them, but wesee that the difference between the acceleration curves for the twopartition cases is very small when we consider a narrow range of values of $\beta_{j}$ (curves (i) and (ii)) and the difference increasesconsiderably when we consider a broad range of values of $\beta_{j}$ (curve (iii) and (iv)). The acceleration attains a much greater valuewhen $\mathcal{D}$ is partitioned into a larger number of subdomains and also becomes negative quicker. The reason for the latter behaviour is that the broad range of values of $\beta_{j}$ makes the third term in (5) gain more prominence when we consider a larger number of subdomains, thus resulting in greater positive acceleration. 


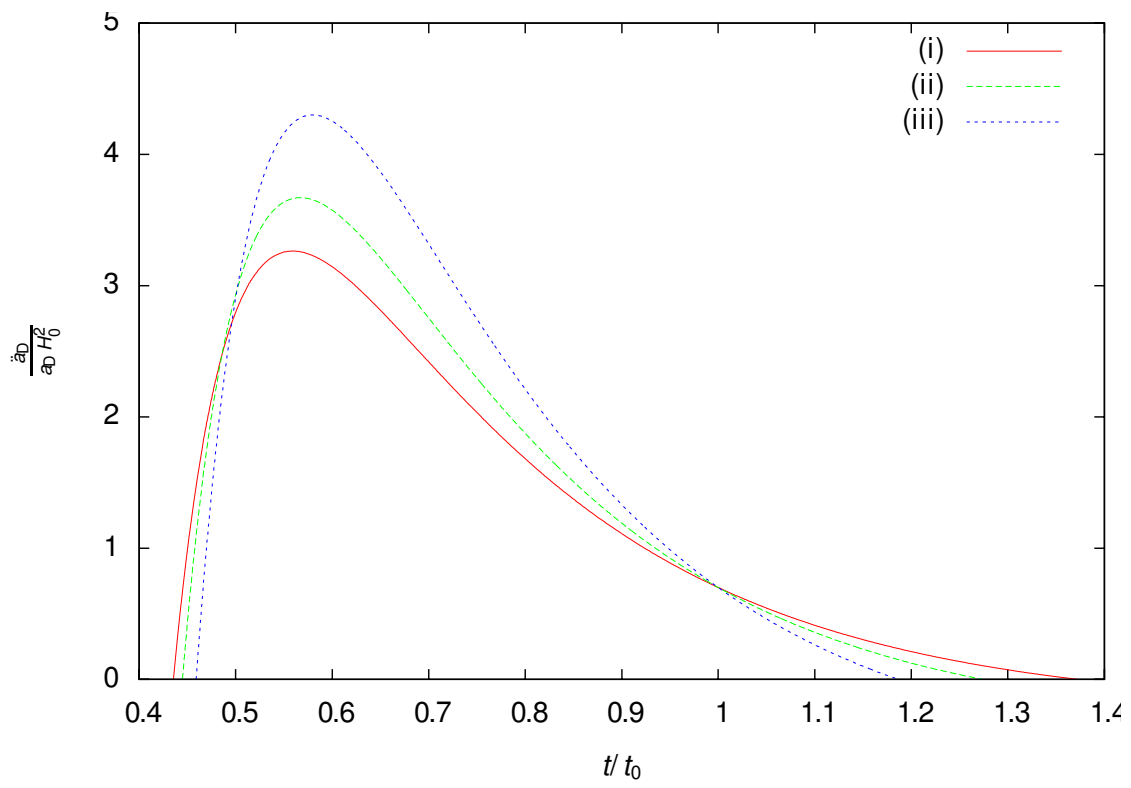

Fig. 3. We plot $\frac{\ddot{a}_{D}}{a_{D} H_{0}^{2}}$ vs. $t / t_{0}$ for various numbers of subdomains. In all the curves we have $\alpha_{j}$ in the range $0.990-0.999$, and $\beta_{j}$ in the range $0.58-0.60$. For curve (i) we consider 100 overdense and underdense subdomains, in (ii) 400 overdense and underdense subdomains, and in (iii) 500 overdense and underdense subdomains each.

In order to see how the global acceleration behaves based on the numberof subdomains we have in Fig. 3 plotted the global acceleration vs.time for three partition cases where we consider (i) 100 overdense and underdense subdomains, (ii) 400 overdense and underdense subdomains and (iii) 500 overdense and underdense subdomains each. For all threecases we have kept the range of values of $\alpha_{j}$ and $\beta_{j}$ the same and taken $\alpha_{j}<1$. It is clearly seen from the plot that the global acceleration increases in magnitude as the numberof subdomains increases, and the maximum is obtained later in time with increase in the number of subdomains. We also see that the acceleration becomes negative faster when the number of subdomains increases.

\section{Summary and Discussions}

The effect of backreaction from inhomogeneities on the global evolution is calculatedwithin the context of the Buchert framework by considering the universe to be divided into multiple underdense and overdense domains, each evolving independently, in order to recreate the real universe more accurately $[4,5,9,12]$. We show that the Buchert framework allows for the 
possibility of the global acceleration vanishing at a finite future time, provided that none of the subdomains accelerate individually (both $\alpha_{j}$ and $\beta_{j}$ are less than 1).

Our analysis shows that if the $\beta_{j}$ parameters are distributed over a narrow range of values and $\alpha_{j}<1$, the global acceleration reaches a greater maximum, when the number of subdomains is larger, showing that the last term in (5), which is always positive, has more prominence for a large number of subdomains. This difference between the accelerations for the two partition cases decreases even more when $\alpha_{j}>1$, because then only the first term in (5) has a negative contribution. However when we consider a broad range of values of $\beta_{j}$, then the difference between the accelerations for the two cases becomes much larger, the acceleration being greater for a larger number of subdomains. The cause for this is attributed to the dominance of the third term in (5) when we have a larger number of subdomains and a broad range of values of $\beta_{j}$.

Our results indicate that backreaction can not only be responsible for the current accelerated expansion, as shown in earlier works [7], but can also cause the acceleration to slow down and even lead to a future decelerated era in some cases. In drawing this conclusion it is not necessary for us to assume that the current acceleration is caused by backreaction, and the acceleration could have been caused by any other mechanism [2].

\section{References}

1. Perlmutter S. (1998). Nature, 391, 51.

2. Sahni V. (2004). Lecture Notesin Physics, Vol. 653.

3. Zalaletdinov R. (1992). Gen. Rel. Grav., 24, 1015.

4. Buchert T. (2000). Gen. Rel. Grav., 32, 105.

5. Buchert T., Carfora M. (2003). Phys. Rev. Lett., 90, 031101.

6. Kolb E. W., Matarrese S., Notari A., Riotto A. (2005). Phys. Rev., D 71, 023524.

7. Rasanen S., Cosmol J. (2004). Astropart. Phys., 0402, 003.

8. Clifton T., Rosquist K., Tavakol R. (2012). Phys. Rev., D 86, 043506.

9. Wiegand A., Buchert T. (2010). Phys. Rev., D 82, 023523.

10. Bose N., Majumdar A. S. (2013). Gen. Rel. Grav., 45, 1971.

11. Bose N., Majumdar A. S. (2011). MNRAS Letters, 418, L45.

12. Buchert T., Carfora M. (2008). Class. Quant. Grav., 25, 195001. 